\title{
Stably Extending Two-Dimensional Bipedal Walking to Three Dimensions
}

\author{
Aaron D. Ames and Robert D. Gregg
}

\begin{abstract}
In this paper we develop a feedback control law that results in stable walking gaits on flat ground for a threedimensional bipedal robotic walker given stable walking gaits for a two-dimensional bipedal robotic walker. This is achieved by combining disparate techniques that have been employed in the bipedal robotic community: controlled symmetries, geometric reduction and hybrid zero dynamics. Controlled symmetries are utilized to obtain stable walking gaits for a two-dimensional bipedal robot walking on flat ground. These are related to walking gaits for a three-dimensional (hipless) bipedal robot through the use of geometric reduction. Finally, these walking gaits in three dimensions are made stable through the use of hybrid zero dynamics.
\end{abstract}

\section{INTRODUCTION}

The central goal of research in bipedal robotic walking is to obtain stable walking gaits, i.e., to prove the existence of stable periodic orbits and/or to develop control laws that create such orbits. Due to the inherent complexity of bipedal robots, many approaches have been employed in order to achieve this goal. While successful in their respective domains of consideration, they often do not extend outside of these domains to obtain a more encompassing solution to the central problem. In this paper we combine these techniques so as to obtain a more satisfying overall solution-this requires a method for "gluing together" these partial solutions. Before proposing our technique, we review preexisting approaches to bipedal walking.

Possibly the first studies in obtaining dynamically stable bipedal walking were concerned with passive twodimensional (2D) bipeds walking down shallow slopes; such walkers have subsequently been well-studied (see [7] and [3] to name a few). It has been shown that for certain shallow slopes, these passive bipeds have stable walking gaits, with no control needed. These walking gaits appear very natural, but unfortunately they only have been shown to exist for a limited class of bipedal robots. For example, the techniques employed by the passive walking community have since been extended to three-dimensional (3D) bipedal walkers (cf. [5]), although the understanding of bipedal walking obtained by this extension seems to be limited since direct control over hip splay is required (which may not be reasonable for certain models). An interesting variant of the passivity paradigm has been applied to a real biped [2].

A. D. Ames is with the Control and Dynamical Systems Department, California Institute of Technology, Pasadena, CA 91125 amesecds.caltech.edu

R. D. Gregg is with the Department of Electrical and Computer Engineering, University of Illinois at Urbana-Champaign, Urbana, IL 61801 rgregg@uiuc. edu
The limitation of the passive approach to bipedal walking is just that-the approach is passive or uncontrolled, thus preventing the application of this idea, for example, to bipeds walking on flat ground (among other varying slopes). This issue was addressed in [10] with the introduction of controlled symmetries. This framework allows for the construction of control laws that translate stable periodic orbits for passive bipeds to stable periodic orbits for actuated bipeds walking on arbitrary slopes. The advantage of this technique is that it exploits the natural symmetries in bipedal walkers to obtain natural and efficient walking gaits. The disadvantage is that it presupposes the existence of passive gaits-these may not exist for certain models of bipedal robots.

The limitations of controlled symmetries highlight the need for a new, more control-oriented method for obtaining walking gaits. The theory that successfully meets this challenge is that of hybrid zero dynamics (see [4] and [8]). This technique uses virtual constraints in the form of output functions to reduce the problem of finding stable periodic orbits to a very low dimensional space where it is tractable. The advantage of this method is that it works for very complex models of bipedal walkers and, in fact, has been implemented on a real robotic system: Rabbit. The disadvantages are that the resulting walking gaits appear unnatural if one does not choose the "correct" virtual constraints, and there are essentially no results on extending this method to 3D bipeds.

The abstract goal of this work is to combine the advantages of the aforementioned techniques, while attempting simultaneously to eliminate the disadvantages, in order to obtain natural stable walking gaits in three dimensions. This requires a new theoretical technique capable of linking these disparate approaches. The authors made a first attempt at obtaining such a framework in [1], where methods from geometric mechanics-specifically, a variant of Routhian reduction termed functional Routhian reduction-were utilized in order to map walking gaits in two dimensions to walking gaits in three dimensions. These methods exploit controlled symmetries (and by association the passive walking paradigm), and so the resulting $3 \mathrm{D}$ gaits have the advantage of being natural. The problem with this approach is that the resulting 3D gaits are stable only from a specific submanifold. Hybrid zero dynamics provide the appropriate framework in which to address this problem, thus linking all of the aforementioned approaches.

The main result of this paper is the construction of a feedback control law that results in stable walking gaits on flat ground for a three-dimensional bipedal robotic walker of a specific form, given stable walking gaits for 
the planar-equivalent two-dimensional bipedal robot. To obtain this result we proceed in the following manner:

Step 1: Consider a passive 2D biped able to walk down a shallow slope.

Step 2: Use controlled symmetries to obtain stable walking gaits on flat ground in two dimensions.

Step 3: Use functional Routhian reduction to obtain a walking gait in three dimensions, stable from a submanifold.

Step 4: Use hybrid zero dynamics to stabilize to the submanifold, thus achieving stable walking gaits in three dimensions.

The final control law resulting from this procedure appears to have the advantage of producing walking gaits that are natural and stable, i.e., it seems to demonstrate the positive aspects of walking gaits obtained from all three of the aforementioned approaches.

\section{HYBRID SYSTEMS}

Hybrid systems are systems that display both continuous and discrete behavior and so bipedal walkers are naturally modeled by systems of this form. This section, therefore, introduces the basic terminology of hybrid systems.

Definition 1: A simple hybrid system ${ }^{1}$ is a tuple:

$$
\mathscr{H}=(D, G, R, f),
$$

where

- $D \subseteq \mathbb{R}^{n}$ is a subset of $\mathbb{R}^{n}$, called the domain,

- $G \subset D$ is subset of $D$ called the guard,

- $R: G \rightarrow D$ is a smooth map called the reset map (or impact equations),

- $f$ is a Lipschitz vector field on $D$, i.e., $\dot{x}=f(x)$.

We also will be interested in studying hybrid control systems.

Definition 2: A simple hybrid control system is a tuple

$$
\mathscr{H} \mathscr{C}=(D, U, G, R, f, g),
$$

where $D, G$ and $R$ are the domain, guard and reset map as introduced in Definition 1 and

- $U \subseteq \mathbb{R}^{k}$ is a set of admissible controls,

- $(f, g)$ is a control system, i.e., $\dot{x}=f(x)+g(x) u$.

Hybrid flows. A hybrid flow is a tuple $\chi^{\mathscr{H}}=(\Lambda, \mathcal{J}, \mathcal{C})$, where

- $\Lambda=\{0,1,2, \ldots\} \subseteq \mathbb{N}$ is an indexing set.

- $\mathcal{J}=\left\{I_{i}\right\}_{i \in \Lambda}$ is a hybrid interval where $I_{i}=\left[\tau_{i}, \tau_{i+1}\right]$ if $i, i+1 \in \Lambda$ and $I_{N-1}=\left[\tau_{N-1}, \tau_{N}\right]$ or $\left[\tau_{N-1}, \tau_{N}\right)$ or $\left[\tau_{N-1}, \infty\right)$ if $|\Lambda|=N, N$ finite. Here, $\tau_{i}, \tau_{i+1}, \tau_{N} \in \mathbb{R}$ and $\tau_{i} \leq \tau_{i+1}$.

\footnotetext{
${ }^{1}$ It is important to note that simple hybrid systems correspond to systems with impulsive effects (see [4] and [8]) and vice versa. Specifically, to a simple hybrid system there is the associated system with impulsive effects of the form:

$$
\Sigma:\left\{\begin{array}{rlrl}
\dot{x} & =f(x) & x & \in D \backslash G \\
x^{+} & =R\left(x^{-}\right) & x^{-} & \in G
\end{array} .\right.
$$
}

- $\mathcal{C}=\left\{c_{i}\right\}_{i \in \Lambda}$ is a collection of integral curves of $f$, i.e., $\dot{c}_{i}(t)=f\left(c_{i}(t)\right)$ for all $i \in \Lambda$.

In addition, we require that for every $i, i+1 \in \Lambda$,

$$
\begin{gathered}
c_{i}\left(\tau_{i+1}\right) \in G, \\
R\left(c_{i}\left(\tau_{i+1}\right)\right)=c_{i+1}\left(\tau_{i+1}\right) .
\end{gathered}
$$

The initial condition for the hybrid flow is $c_{0}\left(\tau_{0}\right)$.

Hybrid periodic orbits. In the context of bipedal robots, we are interested in discussing walking gaits and stable walking gaits - these correspond to hybrid periodic orbits and stable hybrid periodic orbits, respectively.

A hybrid flow $\chi^{\mathscr{H}}=(\Lambda, \mathcal{J}, \mathcal{C})$, of $\mathscr{H}$ is periodic if

- $\Lambda=\mathbb{N}$,

- $\lim _{i \rightarrow \infty} \tau_{i}=\infty$,

- $c_{i}\left(\tau_{i}\right)=c_{i+1}\left(\tau_{i+1}\right)$ for all $i \in \Lambda$.

A hybrid periodic orbit $\mathcal{O} \subset D$ is a subset of $D$ such that

$$
\mathcal{O}=\bigcup_{i \in \mathbb{N}}\left\{c_{i}(t): t \in I_{i}\right\}
$$

for some periodic hybrid flow $\chi^{\mathscr{H}}$

As is standard, denote the distance between a point $x$ and a set $Y$ by $d(x, Y)=\inf _{y \in Y}\|x-y\|$. A hybrid periodic orbit $\mathcal{O}$ is (locally) exponentially stable if there exist constants $M>0, \alpha>0$ and $\delta>0$ such that for all hybrid flows $\chi^{\mathscr{H}}$ with $d\left(c_{0}\left(\tau_{0}\right), \mathcal{O}\right)<\delta$,

$$
d\left(c_{i}(t), \mathcal{O}\right) \leq M e^{-\alpha\left(t-\tau_{0}\right)} d\left(c_{0}\left(\tau_{0}\right), \mathcal{O}\right)
$$

for all $t \in I_{i}$ and $i \in \Lambda$.

\section{BIPEDAL MODELS}

We now introduce the hybrid systems that will be of interest throughout this paper-those modeling a two-dimensional and a three-dimensional bipedal walker without a hip.

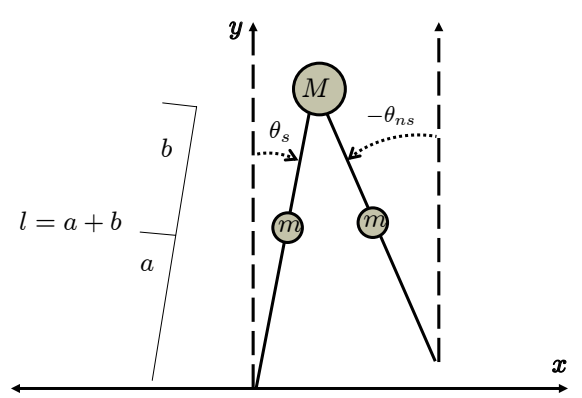

Fig. 1. Two-dimensional bipedal robot.

2D biped model. We begin by introducing a model describing a controlled bipedal robot walking in two dimensions on flat ground (see Figure 1). That is, we explicitly construct the hybrid control system:

$$
\mathscr{H} \mathscr{C}_{2 \mathrm{D}}=\left(D_{2 \mathrm{D}}, U_{2 \mathrm{D}}, G_{2 \mathrm{D}}, R_{2 \mathrm{D}}, f_{2 \mathrm{D}}, g_{2 \mathrm{D}}\right)
$$

describing this system. The techniques utilized in the construction of this hybrid system are further explained in [1]. 
The configuration space ${ }^{2}$ for the $2 \mathrm{D}$ biped is $Q_{2 \mathrm{D}}=\mathbb{R}^{2}$ with coordinates $\theta=\left(\theta_{\mathrm{ns}}, \theta_{\mathrm{s}}\right)^{T}$ where $\theta_{\mathrm{ns}}$ is the angle of the non-stance leg from vertical and $\theta_{\mathrm{s}}$ is the angle of the stance leg from vertical.

The domain and guard are constructed by utilizing the constraint that the non-stance (swing) foot is not allowed to pass through the ground, i.e., by utilizing the unilateral constraint function: $H_{2 \mathrm{D}}(\theta)=\cos \left(\theta_{\mathrm{s}}\right)-\cos \left(\theta_{\mathrm{ns}}\right)$. In particular:

$$
\begin{aligned}
D_{2 \mathrm{D}} & =\left\{\left(\begin{array}{c}
\theta \\
\dot{\theta}
\end{array}\right) \in \mathbb{R}^{4}: H_{2 \mathrm{D}}(\theta) \geq 0\right\} \\
G_{2 \mathrm{D}} & =\left\{\left(\begin{array}{c}
\theta \\
\dot{\theta}
\end{array}\right) \in \mathbb{R}^{4}: H_{2 \mathrm{D}}(\theta)=0,\left(\frac{\partial H_{2 \mathrm{D}}(\theta)}{\partial \theta}\right)^{T} \dot{\theta}<0\right\}
\end{aligned}
$$

We put no restrictions on the set of admissible controls and, therefore, $U_{2 \mathrm{D}}=\mathbb{R}^{2}$.

The reset map $R_{2 \mathrm{D}}$ for $\mathscr{H} \mathscr{C}_{2 \mathrm{D}}$ is computed using the methods outlined in [1] and [4]. In particular, it is given by:

$$
R_{2 \mathrm{D}}(\theta, \dot{\theta})=\left(\begin{array}{c}
S_{2 \mathrm{D}} \theta \\
P_{2 \mathrm{D}}(\theta) \dot{\theta}
\end{array}\right),
$$

where $S_{2 \mathrm{D}}$ and $P_{2 \mathrm{D}}(\theta)$ are given in Table I.

Finally, the dynamics for $\mathscr{H} \mathscr{C}_{2 \mathrm{D}}$ are obtained from the Euler-Lagrange equations in the standard way. Specifically, the Lagrangian describing this system is:

$$
L_{2 \mathrm{D}}(\theta, \dot{\theta})=\frac{1}{2} \dot{\theta}^{T} M_{2 \mathrm{D}}(\theta) \dot{\theta}-V_{2 \mathrm{D}}(\theta),
$$

where $M_{2 \mathrm{D}}(\theta)$ is the inertial matrix and $V_{2 \mathrm{D}}(\theta)$ is the potential energy (these can be found in Table I). The controlled Euler-Lagrange equations yield:

$$
M_{2 \mathrm{D}}(\theta) \ddot{\theta}+C_{2 \mathrm{D}}(\theta, \dot{\theta}) \dot{\theta}+N_{2 \mathrm{D}}(\theta)=B_{2 \mathrm{D}} u,
$$

where $C_{2 \mathrm{D}}(\theta, \dot{\theta})$ is the coriolis matrix and $N_{2 \mathrm{D}}=\frac{\partial V_{2 \mathrm{D}}(\theta)}{\partial \theta}$. In addition, we assume that $B_{2 \mathrm{D}}$ is invertible. These equations yield the control system:

$$
\begin{aligned}
f_{2 \mathrm{D}}(\theta, \dot{\theta}) & =\left(\begin{array}{c}
\dot{\theta} \\
M_{2 \mathrm{D}}(\theta)^{-1}\left(-C_{2 \mathrm{D}}(\theta, \dot{\theta}) \dot{\theta}-N_{2 \mathrm{D}}(\theta)\right)
\end{array}\right) \\
g_{2 \mathrm{D}}(\theta, \dot{\theta}) & =\left(\begin{array}{c}
0_{2 \times 2} \\
M_{2 \mathrm{D}}(\theta)^{-1} B_{2 \mathrm{D}}
\end{array}\right),
\end{aligned}
$$

where $0_{2 \times 2}$ is a $2 \times 2$ matrix of zeros.

We now discuss how a control law can be constructed which yields stable walking gaits (stable periodic orbits) for $\mathscr{H} \mathscr{C}_{2 D}$ utilizing the method of controlled symmetries.

Controlled Symmetries. Controlled symmetries were introduced in [10] in order to shape the potential of bipedal robotic walkers to allow for stable walking gaits on flat ground based on stable walking gaits down a slope (which exist and have been well-studied in [7] and [3]). This is achieved by "rotating the world" via a group action.

\footnotetext{
${ }^{2}$ Technically, the configuration space is given by $Q_{2 \mathrm{D}}=\mathbb{T}^{2}$, the two torus. The motivation for taking the configuration space to be $\mathbb{R}^{2}$ is that for the subset $U$ of $\mathbb{T}^{2}$ containing the angular values of interest, there is a diffeomorphism sending this subset to a subset of $\mathbb{R}^{2}$. Therefore, we simply view the angles as being elements of $\mathbb{R}^{2}$; this allows us to consider coordinates which we can view as being globally defined.
}

Consider the group action $\Psi: \mathbb{S}^{1} \times Q_{2 \mathrm{D}} \rightarrow Q_{2 \mathrm{D}}$ denoted by $\Psi_{\gamma}(\theta):=\left(\theta_{\mathrm{ns}}-\gamma, \theta_{\mathrm{s}}-\gamma\right)^{T}$, for a slope angle $\gamma \in \mathbb{S}^{1}$. Define the following feedback control law:

$$
u=K_{2 \mathrm{D}}^{\gamma}(\theta)=B_{2 \mathrm{D}}^{-1} \frac{\partial}{\partial \theta}\left(V_{2 \mathrm{D}}(\theta)-V_{2 \mathrm{D}}\left(\Psi_{\gamma}(\theta)\right)\right) .
$$

Applying this control law to $\left(f_{2 \mathrm{D}}, g_{2 \mathrm{D}}\right)$ yields the dynamical system: $f_{2 \mathrm{D}}^{\gamma}(\theta, \dot{\theta}):=f_{2 \mathrm{D}}(\theta, \dot{\theta})+g_{2 \mathrm{D}}(\theta, \dot{\theta}) K_{2 \mathrm{D}}^{\gamma}(\theta)$.

The main result of [10] is that there exists ${ }^{3}$ a $\gamma$ such that

$$
\mathscr{H}_{2 \mathrm{D}}^{\mathrm{s}}:=\left(D_{2 \mathrm{D}}, G_{2 \mathrm{D}}, R_{2 \mathrm{D}}, f_{2 \mathrm{D}}^{\gamma}\right)
$$

has a stable walking gait, i.e., an exponentially stable hybrid periodic orbit. Such a periodic orbit can be seen in Figure 3 (in red and blue) with $\gamma=\pi / 50$ radians, $M=10, m=5$, $l=1$ and $a=b=1 / 2$.

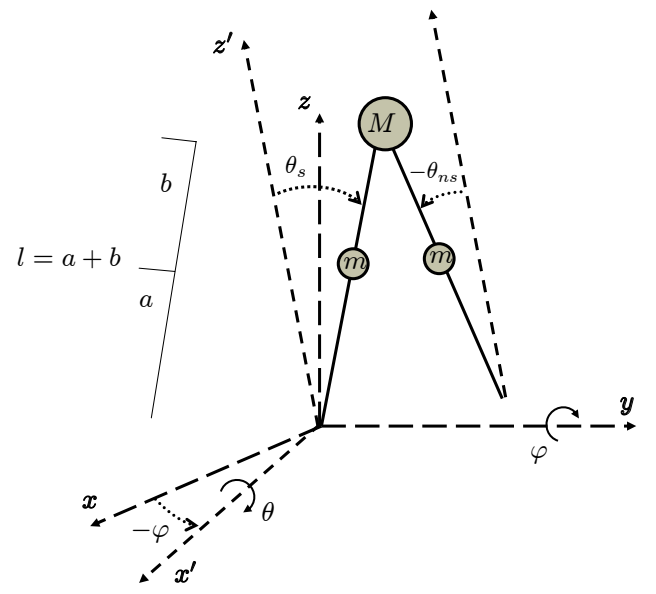

Fig. 2. Three-dimensional bipedal robot.

3D biped model. We now introduce the model describing a controlled bipedal robot walking in three dimensions on flat ground (see Figure 2), i.e., we will explicitly construct the hybrid control system:

$$
\mathscr{H} \mathscr{C}_{3 \mathrm{D}}=\left(D_{3 \mathrm{D}}, U_{3 \mathrm{D}}, G_{3 \mathrm{D}}, R_{3 \mathrm{D}}, f_{3 \mathrm{D}}, g_{3 \mathrm{D}}\right) .
$$

Since this construction is very similar to the construction of $\mathscr{H} \mathscr{C}_{2 \mathrm{D}}$, we will be brief.

The configuration space for the $3 \mathrm{D}$ biped is taken to be $Q_{3 \mathrm{D}}=\mathbb{R}^{3}$, with coordinates $q=\left(\varphi, \theta^{T}\right)^{T}$, where $\varphi$ is the lean (or roll) from vertical and $\theta=\left(\theta_{\mathrm{ns}}, \theta_{\mathrm{s}}\right)^{T}$ as in the 2D biped model.

The guard and domain are constructed from the constraint function: $H_{3 \mathrm{D}}(q)=\cos \left(\theta_{\mathrm{s}}\right)-\cos \left(\theta_{\mathrm{ns}}\right)$, which again gives the scaled height of the non-stance foot above the ground with the implicit assumption that $\varphi \in(-\pi / 2, \pi / 2)$, allowing us to disregard the scaling factor $\cos (\varphi)$ that would have been present. In particular,

$$
\begin{aligned}
& D_{3 \mathrm{D}}=\left\{\left(\begin{array}{c}
q \\
\dot{q}
\end{array}\right) \in \mathbb{R}^{6}: H_{3 \mathrm{D}}(q) \geq 0\right\} \\
& G_{3 \mathrm{D}}=\left\{\left(\begin{array}{c}
q \\
\dot{q}
\end{array}\right) \in \mathbb{R}^{6}: H_{3 \mathrm{D}}(q)=0,\left(\frac{\partial H_{3 \mathrm{D}}(q)}{\partial q}\right)^{T} \dot{q}<0\right\}
\end{aligned}
$$

${ }^{3}$ This $\gamma$ is not unique, but we will pick one once and for all. 


$$
\begin{array}{rlr}
M_{2 \mathrm{D}}(\theta) & =\left(\begin{array}{cc}
\frac{l^{2} m}{4} & -\frac{l^{2} m \cos \left(\theta_{\mathrm{s}}-\theta_{\mathrm{ns}}\right)}{2} \\
-\frac{l^{2} m \cos \left(\theta_{\mathrm{s}}-\theta_{\mathrm{ns}}\right)}{2} & \frac{l^{2} m}{4}+l^{2}(m+M)
\end{array}\right) & S_{2 \mathrm{D}}=\left(\begin{array}{cc}
0 & 1 \\
1 & 0
\end{array}\right) \\
V_{2 \mathrm{D}}(\theta) & =\frac{1}{2} g l\left((3 m+2 M) \cos \left(\theta_{\mathrm{s}}\right)-m \cos \left(\theta_{\mathrm{ns}}\right)\right) & B_{2 \mathrm{D}}=\left(\begin{array}{cc}
-1 & 0 \\
1 & 1
\end{array}\right) \\
P_{2 \mathrm{D}}(\theta) & =\frac{1}{-3 m-4 M+2 m \cos \left(2\left(\theta_{\mathrm{s}}-\theta_{\mathrm{ns}}\right)\right)}\left(\begin{array}{cc}
2 m \cos \left(\theta_{\mathrm{ns}}-\theta_{\mathrm{s}}\right) & m-4(m+M) \cos \left(2\left(\theta_{\mathrm{ns}}-\theta_{\mathrm{s}}\right)\right) \\
m & -2(m+2 M) \cos \left(\theta_{\mathrm{ns}}-\theta_{\mathrm{s}}\right)
\end{array}\right) \\
m_{3 \mathrm{D}}(\theta) & =\frac{1}{8}\left(l^{2}(6 m+4 M)+l^{2}\left(m \cos \left(2 \theta_{\mathrm{ns}}\right)-8 m \cos \left(\theta_{\mathrm{ns}}\right) \cos \left(\theta_{\mathrm{s}}\right)+(5 m+4 M) \cos \left(2 \theta_{\mathrm{s}}\right)\right)\right) \\
V_{3 \mathrm{D}}(\theta, \varphi) & =\frac{V_{2 \mathrm{D}}(\theta) \cos (\varphi)}{6 m+4 M+(5 m+4 M) \cos \left(2 \theta_{\mathrm{ns}}\right)-8 m \cos \left(\theta_{\mathrm{ns}}\right) \cos \left(\theta_{\mathrm{s}}\right)+m \cos \left(2 \theta_{\mathrm{s}}\right)} \\
p_{3 \mathrm{D}}(\theta) & =\frac{-m \cos \left(2 \theta_{\mathrm{ns}}\right)+8(m+M) \cos \left(\theta_{\mathrm{ns}}\right) \cos \left(\theta_{\mathrm{s}}\right)-m\left(2+\cos \left(2 \theta_{\mathrm{s}}\right)\right)}{6 m+4 M \cos (5 m)}
\end{array}
$$

TABLE I

ADDiTIONAL EQUATIONS FOR $\mathscr{H} \mathscr{C}_{2 \mathrm{D}}$ AND $\mathscr{H} \mathscr{C}$ 3D

We again put no restrictions on the set of admissible controls and, therefore, $U_{3 \mathrm{D}}=\mathbb{R}^{3}$.

The reset map $R_{3 \mathrm{D}}$ is given by:

$$
R_{3 \mathrm{D}}(q, \dot{q})=\left(\begin{array}{cccc}
1 & 0 & 0 & 0 \\
0 & S_{2 \mathrm{D}} & 0 & 0 \\
0 & 0 & p_{3 \mathrm{D}}(\theta) & 0 \\
0 & 0 & 0 & P_{2 \mathrm{D}}(\theta)
\end{array}\right)\left(\begin{array}{c}
\varphi \\
\theta \\
\dot{\varphi} \\
\dot{\theta}
\end{array}\right)
$$

where $p_{3 \mathrm{D}}(\theta)$ is given in Table $\mathrm{I}$.

To obtain the dynamics of $\mathscr{H} \mathscr{C}_{3 \mathrm{D}}$ we first note that the Lagrangian describing this system is given by:

$$
L_{3 \mathrm{D}}(q, \dot{q})=\frac{1}{2} \dot{q}^{T} M_{3 \mathrm{D}}(q) \dot{q}-V_{3 \mathrm{D}}(q),
$$

with

$$
M_{3 \mathrm{D}}(q)=\left(\begin{array}{cc}
m_{3 \mathrm{D}}(\theta) & 0 \\
0 & M_{2 \mathrm{D}}(\theta)
\end{array}\right),
$$

where $m_{3 \mathrm{D}}(\theta)$ and $V_{3 \mathrm{D}}(q)$ are given in Table I. Using the controlled Euler-Lagrange equations, the dynamics are given by $M_{3 \mathrm{D}}(q) \ddot{q}+C_{3 \mathrm{D}}(q, \dot{q}) \dot{q}+N_{3 \mathrm{D}}(q)=B_{3 \mathrm{D}} u$, with

$$
B_{3 \mathrm{D}}=\left(\begin{array}{cc}
1 & 0 \\
0 & B_{2 \mathrm{D}}
\end{array}\right) .
$$

These equations yield the control system $\left(f_{3 \mathrm{D}}, g_{3 \mathrm{D}}\right)$ as in the case of the 2D bipedal walker.

\section{IV. $1^{\text {st }}$ CONTROL LAW: RELATING 2D AND 3D Bipeds}

The first step in utilizing stable walking gaits for a $2 \mathrm{D}$ biped to obtain stable walking gaits for a 3D biped is to relate the dynamics of these systems. The method for doing this is the main result of [1], which we summarize here since it forms an integral part of the construction of our second control law - that which yields stable walking gaits in three dimensions given stable walking gaits in two dimensions.

The first control law. The main idea of [1] is that methods from geometric mechanics, and specifically a variant of Routhian reduction [6], can be utilized to relate two- and three-dimensional bipedal walkers satisfying certain assumptions. In order to apply these techniques, it is necessary to shape the potential energy of $L_{3 \mathrm{D}}$, i.e., $V_{3 \mathrm{D}}$, so that it is in a form amenable to the application of hybrid functional
Routhian reduction (see Theorem 2 of [1]). This motivates the introduction of the following control law for $\mathscr{H} \mathscr{C}_{3 \mathrm{D}}$.

Define the feedback control law parameterized by $\alpha \in \mathbb{R}$ :

$$
\begin{aligned}
u & =K_{3 \mathrm{D}}^{\alpha}(q) \\
& :=B_{3 \mathrm{D}}^{-1} \frac{\partial}{\partial q}\left(V_{3 \mathrm{D}}(q)-V_{2 \mathrm{D}}\left(\Psi_{\gamma}(\theta)\right)+\frac{1}{2} \frac{\alpha^{2} \varphi^{2}}{m_{3 \mathrm{D}}(\theta)}\right),
\end{aligned}
$$

with $\gamma$ as in (1). Applying this control law to the control system $\left(f_{3 \mathrm{D}}, g_{3 \mathrm{D}}\right)$ yields the dynamical system:

$$
f_{3 \mathrm{D}}^{\alpha}(q, \dot{q}):=f_{3 \mathrm{D}}(q, \dot{q})+g_{3 \mathrm{D}}(q, \dot{q}) K_{3 \mathrm{D}}^{\alpha}(q)
$$

whence we obtain a hybrid system:

$$
\mathscr{H}_{3 \mathrm{D}}^{\alpha}:=\left(D_{3 \mathrm{D}}, G_{3 \mathrm{D}}, R_{3 \mathrm{D}}, f_{3 \mathrm{D}}^{\alpha}\right) .
$$

The main result of [1] is the following:

Theorem 1: $\chi^{\mathscr{H}_{3 \mathrm{D}}^{\alpha}}=\left(\Lambda, \mathcal{J},\left\{\left(\theta_{i}, \dot{\theta}_{i}, \varphi_{i}, \dot{\varphi}_{i}\right)\right\}_{i \in \Lambda}\right)$ is a hybrid flow of $\mathscr{H}_{3 \mathrm{D}}^{\alpha}$ with

$$
\dot{\varphi}_{0}\left(\tau_{0}\right)=-\frac{\alpha \varphi_{0}\left(\tau_{0}\right)}{m_{3 \mathrm{D}}\left(\theta_{0}\left(\tau_{0}\right)\right)},
$$

if and only if $\chi^{\mathscr{H}_{2 \mathrm{D}}^{\mathrm{s}}}=\left(\Lambda, \mathcal{J},\left\{\theta_{i}, \dot{\theta}_{i}\right\}_{i \in \Lambda}\right)$ is a hybrid flow of $\mathscr{H}_{2 \mathrm{D}}^{\mathrm{s}}$ and $\left\{\left(\varphi_{i}, \dot{\varphi}_{i}\right)\right\}_{i \in \Lambda}$ satisfies:

$$
\begin{gathered}
\dot{\varphi}_{i}(t)=-\frac{\alpha \varphi_{i}(t)}{m_{3 \mathrm{D}}\left(\theta_{i}(t)\right)}, \\
\varphi_{i+1}\left(\tau_{i+1}\right)=\varphi_{i}\left(\tau_{i+1}\right) .
\end{gathered}
$$

Implications of Theorem 1. We now devote some attention to discussing the implications of Theorem 1 and what it does, and does not, imply about the behavior of $\mathscr{H}_{3 \mathrm{D}}^{\alpha}$.

Let $\iota_{0}: \mathbb{R}^{4} \rightarrow \mathbb{R}^{6}$ be the inclusion given by $\iota_{0}(\theta, \dot{\theta})=$ $\left(0, \theta^{T}, 0, \dot{\theta}^{T}\right)^{T}$. The first implication of Theorem 1 is the following:

Corollary 1: If $\mathcal{O}_{2 \mathrm{D}}$ is a hybrid periodic orbit of $\mathscr{H}_{2 \mathrm{D}}^{\mathrm{s}}$, then $\mathcal{O}_{3 \mathrm{D}}:=\iota_{0}\left(\mathcal{O}_{2 \mathrm{D}}\right)$ is a hybrid periodic orbit of $\mathscr{H}_{3 \mathrm{D}}^{\alpha}$.

Therefore, if $\mathscr{H}_{2 \mathrm{D}}^{\mathrm{s}}$ has a walking gait in two dimensions, then $\mathscr{H}_{3 \mathrm{D}}^{\alpha}$ has a walking gait in three dimensions. Herein lies the importance of Theorem 1, although one immediately sees its limitations - nothing can be said about the stability of this walking gait (in the sense of local exponential stability).

Utilizing (3) and (4), we can obtain an understanding of the nature of the periodic orbit $\mathcal{O}_{3 \mathrm{D}}$. If $\chi^{\mathscr{H}_{3 \mathrm{D}}^{\alpha}}$ is a hybrid flow of $\mathscr{H}_{3 \mathrm{D}}^{\alpha}$ satisfying (3), then Theorem 1 implies that (4) holds. Therefore, the walker stabilizes to the "upright" 


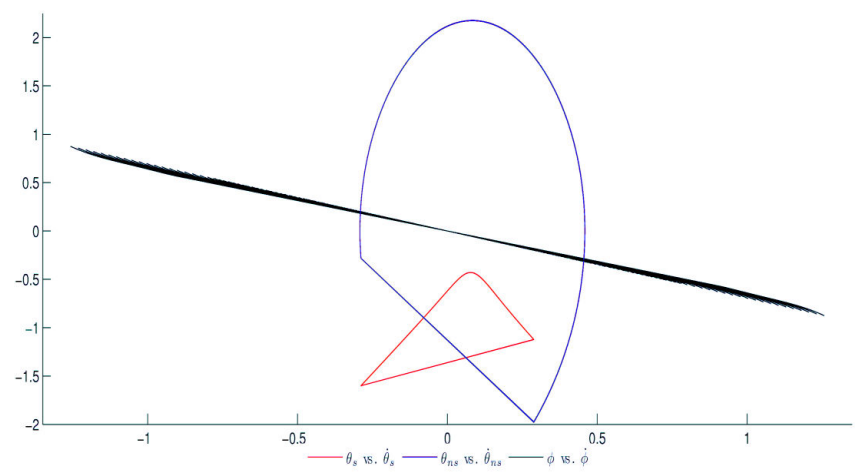

Fig. 3. Phase portraits for different walking gaits obtained by varying $\varphi\left(\tau_{0}\right)$ and $\dot{\varphi}\left(\tau_{0}\right)$ and keeping the initial values of $\theta$ and $\dot{\theta}$ constant. The black region shows values of $\varphi\left(\tau_{0}\right)$ and $\dot{\varphi}\left(\tau_{0}\right)$ that result in convergence to $\mathcal{O}_{3 \mathrm{D}}$.

position. This is because the roll, $\varphi$, will tend to zero as time goes to infinity since (4) essentially defines a stable linear system $\dot{\varphi}=-\alpha \varphi$ when $\alpha>0$, because $m_{3 \mathrm{D}}\left(\theta_{i}(t)\right)>0$ by the positive definiteness of $M_{3 \mathrm{D}}$. Thus the lateral and sagittal dynamics effectively have been decoupled. We conclude that the periodic orbit $\mathcal{O}_{3 \mathrm{D}}$ is "stable" from initial conditions satisfying (3); a plot of this set of initial conditions can be seen in Figure 3. Unfortunately, the dynamics are unstable for initial conditions not satisfying (3). The goal of the second control law, to be introduced in the next section, is to render the periodic orbit stable.

\section{V. $2^{\text {nd }}$ Control LaW: Stable Walking Gaits in 3D}

In this section, we introduce our second control law with the goal of stabilizing to the surface from which trajectories converge to the periodic orbit $\mathcal{O}_{3 \mathrm{D}}$-that is, we render $\mathcal{O}_{3 \mathrm{D}}$ stable. To achieve this goal, we utilize the main result of [8].

A new hybrid control system. Before introducing the second control law, we define a new hybrid system that implicitly utilizes the first control law (as introduced in the previous section). Specifically, let

$$
\mathscr{H} \mathscr{C}_{3 \mathrm{D}}^{\alpha}=\left(D_{3 \mathrm{D}}, \mathbb{R}, G_{3 \mathrm{D}}, R_{3 \mathrm{D}}, f_{3 \mathrm{D}}^{\alpha}, g_{3 \mathrm{D}}^{\alpha}\right)
$$

where $D_{3 \mathrm{D}}, G_{3 \mathrm{D}}$ and $R_{3 \mathrm{D}}$ are defined as for $\mathscr{H} \mathscr{C}_{3 \mathrm{D}}$ (defined in Section III). The control system $\left(f_{3 \mathrm{D}}^{\alpha}, g_{3 \mathrm{D}}^{\alpha}\right)$ is obtained by applying the control law

$$
u=K_{3 \mathrm{D}}^{\alpha}(q)+\left(\begin{array}{ccc}
1 & 0 & 0
\end{array}\right)^{T} v
$$

to $\mathscr{H} \mathscr{C}_{3 \mathrm{D}}$, where $v \in \mathbb{R}$. In particular, $f_{3 \mathrm{D}}^{\alpha}(q, \dot{q})$ is given as in (2) and

$$
g_{3 \mathrm{D}}^{\alpha}(q, \dot{q})=\left(\begin{array}{llllll}
0 & 0 & 0 & \frac{1}{m_{3 \mathrm{D}}(\theta)} & 0 & 0
\end{array}\right)^{T} .
$$

The second control law. Motivated by our desire to satsify (4), let

$$
h(q, \dot{q}):=\dot{\varphi}+\frac{\alpha \varphi}{m_{3 \mathrm{D}}(\theta)}
$$

The main idea in the construction of the second control law is that we would like to drive $h(q, \dot{q})$ to zero, i.e., we would like to drive the system to the surface

$$
z=\left\{\left(\begin{array}{c}
q \\
\dot{q}
\end{array}\right) \in \mathbb{R}^{6}: h(q, \dot{q})=0\right\},
$$

wherein the first control law introduced in the previous section should take over (roughly speaking, the surface $z$ is the black region in Figure 3). From Theorem 1, we know that solutions will then converge to the periodic orbit $\mathcal{O}_{3 \mathrm{D}}$. We thus will have rendered this periodic orbit stable.

With this in mind, and motivated by the standard method for driving an output to zero in a nonlinear control system (see [9]), we define the following feedback control law:

$$
\begin{aligned}
v & =K_{3 \mathrm{D}}^{\alpha, \epsilon}(q, \dot{q}) \\
& :=\frac{-1}{L_{g_{3 \mathrm{D}}^{\alpha}} h(q, \dot{q})}\left(L_{f_{3 \mathrm{D}}^{\alpha}} h(q, \dot{q})+\frac{1}{\epsilon} h(q, \dot{q})\right),
\end{aligned}
$$

where $L_{g_{3 \mathrm{D}}^{\alpha}} h(q, \dot{q})$ is the Lie derivative of $h$ with respect to $g_{3 \mathrm{D}}^{\alpha}$ and $L_{f_{3 \mathrm{D}}^{\alpha}} h(q, \dot{q})$ is the Lie derivative of $h$ with respect to $f_{3 \mathrm{D}}^{\alpha}$. Note that we know $K_{3 \mathrm{D}}^{\alpha, \epsilon}(q, \dot{q})$ is well-defined since

$$
L_{g_{3 \mathrm{D}}^{\alpha}} h(q, \dot{q})=\frac{1}{m_{3 \mathrm{D}}(\theta)},
$$

and $m_{3 \mathrm{D}}(\theta)>0$ by the positive definiteness of $M_{3 \mathrm{D}}$.

Utilizing the feedback control law $K_{3 \mathrm{D}}^{\alpha, \epsilon}(q, \dot{q})$, we obtain a hybrid system:

$$
\mathscr{H}_{3 \mathrm{D}}^{\alpha, \epsilon}:=\left(D_{3 \mathrm{D}}, G_{3 \mathrm{D}}, R_{3 \mathrm{D}}, f_{3 \mathrm{D}}^{\alpha, \epsilon}\right),
$$

where $f_{3 \mathrm{D}}^{\alpha, \epsilon}(q, \dot{q}):=f_{3 \mathrm{D}}^{\alpha}(q, \dot{q})+g_{3 \mathrm{D}}^{\alpha}(q, \dot{q}) K_{3 \mathrm{D}}^{\alpha, \epsilon}(q, \dot{q})$. We now have the necessary framework in which to introduce the main result of this paper.

Theorem 2: If $\mathscr{H}_{2 \mathrm{D}}^{\mathrm{s}}$ has a locally exponentially stable hybrid periodic orbit $\mathcal{O}_{2 \mathrm{D}}$ such that

- $\left(\begin{array}{c}\theta^{*} \\ \dot{\theta}^{*}\end{array}\right)=\mathcal{O}_{2 \mathrm{D}} \cap G_{2 \mathrm{D}}$ is a singleton,

- $L_{f_{2 \mathrm{D}}^{\mathrm{s}}} H_{2 \mathrm{D}}\left(\theta^{*}, \dot{\theta}^{*}\right) \neq 0$,

then there exists a $\delta>0$ such that $\mathcal{O}_{3 \mathrm{D}}=\iota_{0}\left(\mathcal{O}_{2 \mathrm{D}}\right)$ is $a$ locally exponentially stable hybrid periodic orbit of $\mathscr{H}_{3 \mathrm{D}}^{\alpha, \epsilon}$ for all $\alpha>0$ and all $\epsilon$ such that $\delta>\epsilon>0$.

This theorem essentially follows from the previous results of this paper coupled with the Main Theorem of [8]. To see this, we first must introduce a global diffeomorphism that makes the structure of $\mathscr{H}_{3 \mathrm{D}}^{\alpha, \epsilon}$ more transparent. We then provide a proof of Theorem 2 .

The coordinate transformation. Consider the following global diffeomorphism:

$$
\Phi(q, \dot{q})=\left(\begin{array}{c}
h(q, \dot{q}) \\
\theta \\
\dot{\varphi} \\
\dot{\theta}
\end{array}\right)=:\left(\begin{array}{l}
\eta \\
z
\end{array}\right),
$$

where $\eta \in \mathbb{R}$ and $z=\left(\theta^{T}, \dot{\varphi}, \dot{\theta}^{T}\right)^{T} \in \mathbb{R}^{5}$. It is easy to see that this is in fact a global diffeomorphism since it clearly is smooth and has a smooth inverse:

$$
\Phi^{-1}(\eta, z)=\left(\frac{m_{3 \mathrm{D}}\left(z_{1}, z_{2}\right)}{\alpha}\left(\eta-z_{3}\right)\right),
$$


where it follows from the definition of $z$ that $z_{1}=\theta_{\mathrm{ns}}$, $z_{2}=\theta_{\mathrm{s}}$ and $z_{3}=\dot{\varphi}$. Note that in this new coordinate system the surface $z$ becomes:

$$
\widetilde{z}=\left\{\left(\begin{array}{c}
\eta \\
z
\end{array}\right) \in \mathbb{R}^{6}: \eta=0\right\} .
$$

Transforming $\mathscr{H}_{3 \mathrm{D}}^{\alpha, \epsilon}$. We now proceed to transform $\mathscr{H}_{3 \mathrm{D}}^{\alpha, \epsilon}$ to this new coordinate system; we will denote the transformed system by:

$$
\widetilde{\mathscr{H}}_{3 \mathrm{D}}^{\alpha, \epsilon}=\left(\widetilde{D}_{3 \mathrm{D}}, \widetilde{G}_{3 \mathrm{D}}, \widetilde{R}_{3 \mathrm{D}}, \widetilde{f}_{3 \mathrm{D}}^{\alpha, \epsilon}\right) .
$$

The domain and guard for the transformed system and the original system are the same:

$$
\widetilde{D}_{3 \mathrm{D}}=\Phi\left(D_{3 \mathrm{D}}\right)=D_{3 \mathrm{D}}, \quad \widetilde{G}_{3 \mathrm{D}}=\Phi\left(G_{3 \mathrm{D}}\right)=G_{3 \mathrm{D}},
$$

by the definition of $D_{3 \mathrm{D}}$ and $G_{3 \mathrm{D}}$, i.e., by the fact that $H_{3 \mathrm{D}}$ is independent of $\varphi$. In particular, if we let

$$
\widetilde{H}_{3 \mathrm{D}}(z)=\cos \left(z_{2}\right)-\cos \left(z_{1}\right),
$$

then it follows that:

$$
\begin{aligned}
& \widetilde{D}_{3 \mathrm{D}}=\left\{\left(\begin{array}{c}
\eta \\
z
\end{array}\right) \in \mathbb{R}^{6}: \widetilde{H}_{3 \mathrm{D}}(z) \geq 0\right\} \\
& \widetilde{G}_{3 \mathrm{D}}=\left\{\left(\begin{array}{c}
\eta \\
z
\end{array}\right) \in \mathbb{R}^{6}: \begin{array}{c}
\widetilde{H}_{3 \mathrm{D}}(z)=0, \\
\sin \left(z_{1}\right) z_{4}-\sin \left(z_{2}\right) z_{5}<0
\end{array}\right\}
\end{aligned}
$$

The reset map for the transformed system is given by:

$$
\begin{aligned}
& \widetilde{R}_{3 \mathrm{D}}(\eta, z)=\Phi \circ R_{3 \mathrm{D}} \circ \Phi^{-1}(\eta, z) \\
& =\left(\begin{array}{c}
p_{3 \mathrm{D}}\left(z_{1}, z_{2}\right) \eta \\
S_{2 \mathrm{D}}\left(\begin{array}{c}
z_{1} \\
z_{2}
\end{array}\right) \\
p_{3 \mathrm{D}}\left(z_{1}, z_{2}\right) z_{3} \\
P_{2 \mathrm{D}}\left(z_{1}, z_{2}\right)\left(\begin{array}{c}
z_{4} \\
z_{5}
\end{array}\right)
\end{array}\right)
\end{aligned}
$$

since

$$
p_{3 \mathrm{D}}\left(z_{1}, z_{2}\right)=\frac{m_{3 \mathrm{D}}\left(z_{1}, z_{2}\right)}{m_{3 \mathrm{D}}\left(z_{2}, z_{1}\right)}
$$

when $\widetilde{H}_{3 \mathrm{D}}(z)=0$. Finally, the transformed vector field is given by

$$
\tilde{f}_{3 \mathrm{D}}^{\alpha, \epsilon}(\eta, z)=D \Phi\left(\Phi^{-1}(\eta, z)\right) f_{3 \mathrm{D}}^{\alpha, \epsilon}\left(\Phi^{-1}(\eta, z)\right),
$$

where $D \Phi(q, \dot{q})$ is the Jacobian of $\Phi$ (evaluated at $(q, \dot{q}))$. In particular, because of the construction of $K_{3 \mathrm{D}}^{\alpha, \epsilon}$,

$$
\dot{\eta}=-\frac{1}{\epsilon} \eta
$$

We now prove Theorem 2 .

Proof: In order to apply the main result of [8], we must verify that assumptions (H1.1)-(H1.6) and (H2.1)-(H2.6) hold inasmuch as they are needed ${ }^{4}$ in the proof of the Main Theorem of [8]. It is immediately obvious that most of the assumptions hold, so we only comment on the assumptions

\footnotetext{
${ }^{4}$ That is, these assumptions do not hold as they are stated in this paper, but they are violated to such a small degree that it does not affect the main result of this work.
}

that require some verification (space constraints prevent us from including a more detailed proof).

(H1.1)-(H1.6) hold with the exception that $\widetilde{D}_{3 \mathrm{D}}$ is not an open set and $\widetilde{G}_{3 \mathrm{D}} \neq \widetilde{H}_{3 \mathrm{D}}^{-1}(0)$. Both of these violations do not affect the Main Result of [8]. For example, $\widetilde{G}_{3 \mathrm{D}} \subset \widetilde{H}_{3 \mathrm{D}}^{-1}(0)$ and is a smooth manifold of codimension one. Therefore, it can be viewed as the zero-level set of $\widetilde{H}_{3 \mathrm{D}}$ in the region of interest, i.e., in a neighborhood of the periodic orbit $\mathcal{O}_{3 \mathrm{D}}$.

(H2.1): In our case, the $z$-dynamics are not independent of $\epsilon$ as is required by (H2.1) in [8]; despite this, the axiom is sufficiently satisfied inasmuch as it is needed in this paper. Specifically, $\widetilde{f}_{3 \mathrm{D}}^{\alpha, \epsilon}(0, z)$ is independent of $\epsilon$ and Theorem 1 implies that $\widetilde{z}$ is invariant under the continuous part of the model, i.e., the dynamics on $z$ are given by $f_{3 \mathrm{D}}^{\alpha}$ which is independent of $\epsilon$.

(H2.2): Consider the map $\Upsilon: \widetilde{D}_{3 \mathrm{D}} \rightarrow \mathbb{R}^{2}$ with

$$
\Upsilon(\eta, z):=\left(\begin{array}{c}
\eta \\
\widetilde{H}_{3 \mathrm{D}}(z)
\end{array}\right) \text {. }
$$

It is easy to verify that $\operatorname{rank}(D \Upsilon(\eta, z))=2$ when restricted to $\widetilde{G}_{3 \mathrm{D}}$, and so $\widetilde{G}_{3 \mathrm{D}} \cap \widetilde{z}$ is a $(n-2)$-dimensional submanifold of $\widetilde{D}_{3 \mathrm{D}}$. In addition, it follows from (5) that

$$
\widetilde{R}_{3 \mathrm{D}}\left(\widetilde{G}_{3 \mathrm{D}} \cap \widetilde{z}\right) \subset \widetilde{z}
$$

since $\eta=0$ implies that $p_{3 \mathrm{D}}\left(z_{1}, z_{2}\right) \eta=0$.

$(\mathrm{H} 2.3)$ : Since $\mathcal{O}_{3 \mathrm{D}}=\iota_{0}\left(\mathcal{O}_{2 \mathrm{D}}\right)$, clearly $\mathcal{O}_{3 \mathrm{D}} \subset z$.

(H2.4): Due to the difference in the notion of solution between [8] and this paper, we need not take the closure of $\mathcal{O}_{3 \mathrm{D}}$ (since it is already closed). Now a simple calculation shows that:

$\left(\begin{array}{c}\theta^{*} \\ \dot{\theta}^{*}\end{array}\right)=\mathcal{O}_{2 \mathrm{D}} \cap G_{2 \mathrm{D}} \quad \Rightarrow \quad \iota_{0}\left(\begin{array}{c}\theta^{*} \\ \dot{\theta}^{*}\end{array}\right)=\mathcal{O}_{3 \mathrm{D}} \cap G_{3 \mathrm{D}} \cap z$ and so $\Phi\left(\mathcal{O}_{3 \mathrm{D}}\right) \cap \widetilde{G}_{3 \mathrm{D}} \cap \widetilde{z}=\Phi\left(\iota_{0}\left(\theta^{*}, \dot{\theta}^{*}\right)\right)$ is a singleton.

(H2.5): Due to the specific form of $f_{3 \mathrm{D}}^{\alpha, \epsilon}$, the fact that $H_{3 \mathrm{D}}$ is independent of $\varphi$ and $\dot{\varphi}$ and the fact that $\Phi$ leaves $\theta$ and $\dot{\theta}$ fixed, it follows that:

$$
\begin{aligned}
L_{f_{2 \mathrm{D}}^{\mathrm{s}}} H_{2 \mathrm{D}}\left(\theta^{*}, \dot{\theta}^{*}\right) & =L_{f_{3 \mathrm{D}}^{\alpha, \epsilon}} H_{3 \mathrm{D}}\left(\iota_{0}\left(\theta^{*}, \dot{\theta}^{*}\right)\right) \\
& =L_{\widetilde{f}_{3 \mathrm{D}}^{\alpha, \epsilon}} \widetilde{H}_{3 \mathrm{D}}\left(\Phi\left(\iota_{0}\left(\theta^{*}, \dot{\theta}^{*}\right)\right)\right) .
\end{aligned}
$$

Therefore,

$$
L_{f_{2 \mathrm{D}}^{\mathrm{s}}} H_{2 \mathrm{D}}\left(\theta^{*}, \dot{\theta}^{*}\right) \neq 0 \Rightarrow L_{\widetilde{f}_{3 \mathrm{D}}^{\alpha, \epsilon}} \widetilde{H}_{3 \mathrm{D}}\left(\Phi\left(\iota_{0}\left(\theta^{*}, \dot{\theta}^{*}\right)\right)\right) \neq 0 .
$$

(H2.6): Follows from (6).

Simulation results. To demonstrate the effectiveness of the proposed control law and the usefulness of Theorem 2, we provide some simulation results. For these simulations, we begin with a numerically-computed periodic orbit $\mathcal{O}_{2 \mathrm{D}}$ for $\mathscr{H}_{2 \mathrm{D}}^{\mathrm{s}}$; this periodic orbit can be seen in Figure 3 (in red and blue). It can be verified numerically that this periodic orbit is stable and satisfies the assumptions of Theorem 2; in particular,

$$
\left(\begin{array}{c}
\theta^{*} \\
\dot{\theta}^{*}
\end{array}\right)=\mathcal{O}_{2 \mathrm{D}} \cap G_{2 \mathrm{D}} \approx\left(\begin{array}{c}
-0.2884 \\
0.2884 \\
-1.6009 \\
-1.9762
\end{array}\right) .
$$




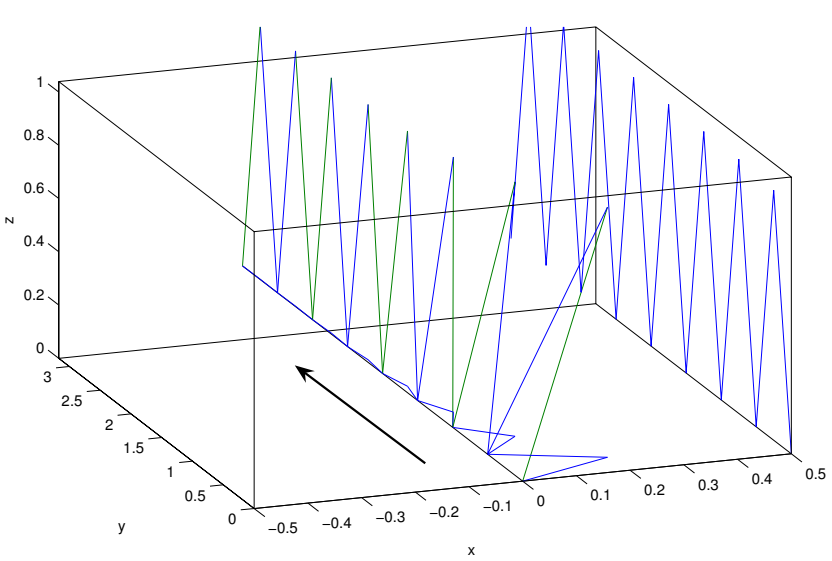

Fig. 4. A walking gait for the three-dimensional biped.

Therefore, by this theorem, we know that $\mathcal{O}_{3 \mathrm{D}}=\iota_{0}\left(\mathcal{O}_{2 \mathrm{D}}\right)$ is a stable periodic orbit of $\mathscr{H}_{3 \mathrm{D}}^{\alpha, \epsilon}$ for $\alpha>0$ and for sufficiently small $\epsilon>0$.

We first note that the stability of $\mathcal{O}_{3 \mathrm{D}}$ implies the existence of stable walking gaits. An example of a specific walking gait can be seen in Figure 4 for $\epsilon=1 / 15$. For this walking gait, the biped exponentially converges to the "upright" position as the theory would indicate. This exponential convergence can be better visualized in Figure 5, where $\varphi\left(\tau_{0}\right)$ is varied while $\dot{\varphi}\left(\tau_{0}\right)=0, \theta\left(\tau_{0}\right)=\theta^{*}$ and $\dot{\theta}\left(\tau_{0}\right)=\dot{\theta}^{*}$ are held constant.

While we know that the periodic orbit $\mathcal{O}_{3 \mathrm{D}}$ is locally exponentially stable by Theorem 2 , this theorem does not yield any information about the radius of convergence of this periodic orbit. A better understanding can be obtained through simulation. Since the region of stability for $\mathcal{O}_{2 \mathrm{D}}$ has been well-studied (see [3]), we are interested in the region of stability of $\mathcal{O}_{3 \mathrm{D}}$ in the $(\varphi, \dot{\varphi})$-plane. We therefore simulate hybrid flows of $\mathscr{H}_{3 \mathrm{D}}^{\alpha, \epsilon}$ while fixing $\theta\left(\tau_{0}\right)=\theta^{*}$ and $\dot{\theta}\left(\tau_{0}\right)=\dot{\theta}^{*}$ and varying $\varphi\left(\tau_{0}\right)$ and $\dot{\varphi}\left(\tau_{0}\right)$. The resulting behavior can be seen in Figure 6. In this figure, the red and blue regions are a result of perturbations in the $(\theta, \dot{\theta})$ dynamics that occur when initial conditions are chosen off of the surface $z$. The lateral and sagittal dynamics are no longer decoupled because Theorem 1 does not hold for these values. For these initial conditions off of the surface $z$, the second control law drives the $(\varphi, \dot{\varphi})$-dynamics to this surface as the figure indicates. Finally, we note that this simulation result seems to imply that the region of convergence of $\mathcal{O}_{3 \mathrm{D}}$ is fairly large, indicating that the proposed law can effectively stabilize the biped from a large set of initial conditions.

\section{CONCLUSION}

In this paper, a method was presented for stably extending two-dimensional walking gaits to three dimensions using geometric reduction. The techniques utilized to obtain this result also can be applied to three-dimensional bipeds with a hip; this will be the subject of future papers. Finally, in this paper, the assumption of full actuation was necessaryextensions to the case of underactuated bipedal robots is a very interesting and promising research direction.

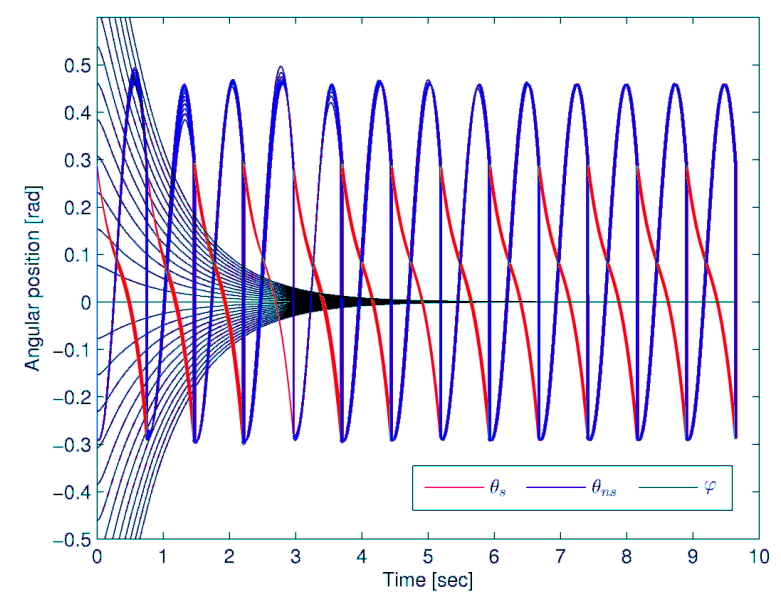

Fig. 5. The exponential convergence of $\varphi$ for different values of $\varphi\left(\tau_{0}\right)$.

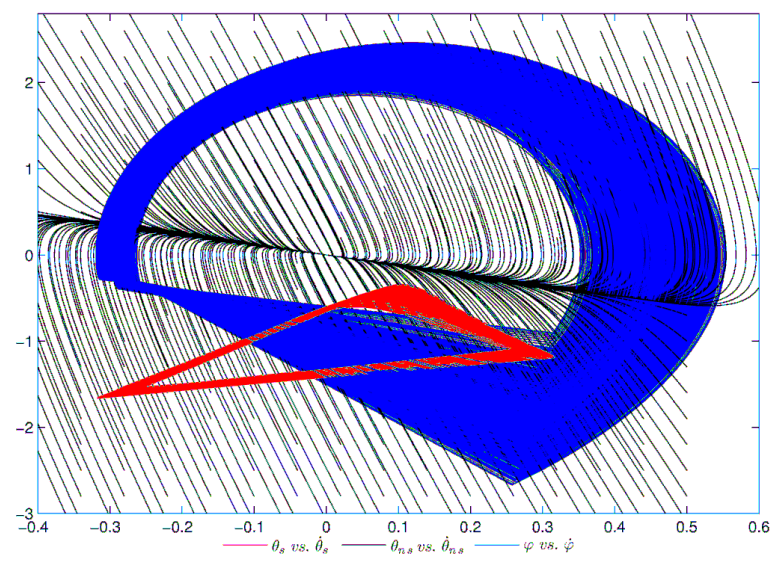

Fig. 6. The convergent behavior of $\mathcal{O}_{3 \mathrm{D}}$ for different initial values of $\varphi$ and $\dot{\varphi}$.

\section{REFERENCES}

[1] A. D. Ames, R. D. Gregg, E. D. B. Wendel, and S. Sastry, "Towards the geometric reduction of controlled three-dimensional robotic bipedal walkers," in 3rd Workshop on Lagrangian and Hamiltonian Methods for Nonlinear Control (LHMNLC'O6), Nagoya, Japan, 2006.

[2] S. H. Collins, M. Wisse, and A. Ruina, "A 3-D passive dynamic walking robot with two legs and knees," International Journal of Robotics Research, vol. 20, pp. 607-615, 2001.

[3] A. Goswami, B. Thuilot, and B. Espiau, "Compass-like biped robot part I : Stability and bifurcation of passive gaits," 1996, rapport de recherche de l'INRIA.

[4] J. Grizzle, G. Abba, and F. Plestan, "Asymptotically stable walking for biped robots: Analysis via systems with impulse effects," IEEE Transactions on Automatic Control, vol. 46, no. 1, pp. 51-64, 2001.

[5] A. D. Kuo, "Stabilization of lateral motion in passive dynamic walking," International Journal of Robotics Research, vol. 18, no. 9, pp. 917-930, 1999.

[6] J. E. Marsden and T. S. Ratiu, Introduction to Mechanics and Symmetry, ser. Texts in Applied Mathematics. Springer, 1999, vol. 17.

[7] T. McGeer, "Passive dynamic walking," International Journal of Robotics Research, vol. 9, no. 2, pp. 62-82, 1990.

[8] B. Morris and J. W. Grizzle, "A restricted Poincaré map for determining exponentially stable periodic orbits in systems with impulse effects: Application to bipedal robots," in Conference on Decision and Control, Seville, Spain, 2006.

[9] S. Sastry, Nonlinear Systems: Analysis, Stability and Control. Springer-Verlag, 1999.

[10] M. W. Spong and F. Bullo, "Controlled symmetries and passive walking," IEEE Transactions on Automatic Control, vol. 50, no. 7, pp. 1025-1031, 2005. 